\title{
Functional Relations in Stokes Multipliers and Solvable Models related to $U_{q}\left(A_{n}^{(1)}\right)$
}

\author{
J. Suzuki* \\ Department of Physics, Faculty of Science \\ Shizuoka University, \\ Ohya 836, Shizuoka, \\ Japan
}

October 1999

\begin{abstract}
Recently, Dorey and Tateo have investigated functional relations among Stokes multipliers for a Schrödinger equation (second order differential equation) with a polynomial potential term in view of solvable models. Here we extend their studies to a restricted case of $n+1$-th order linear differential equations.
\end{abstract}

*e-mail: sjsuzuk@ipc.shizuoka.ac.jp 


\section{Introduction}

In remarkable papers [1, [], Dorey and Tateo find marvelous connections between a Schrödinger equation with a polynomial potential term and solvable models. The success integrates two ingredients. The exact WKB analysis [3]-[10] is on the one side. The development in studies of solvable models and of the solvability structure in (perturbed) CFT [11, 12, 13, 14] via the thermodynamic Bethe ansatz [16, 17] and nonlinear integral equations is on the other 18]-22].

In view of the eigenvalue problem in quantum mechanics, $\mathcal{H} \Psi=E \Psi$, the quantity of interest is the spectral determinant, $\operatorname{det}(\mathcal{H}-E)$. For the potential term $x^{2 M}$ with $M=$ integer, it coincides with the "vacuum expectation value" of a particular member of a fusion transfer matrices in a certain field theory possessing $U_{q}\left(\widehat{\mathfrak{s l}_{2}}\right)$ [1, 23]. The rest of members in the fusion hierarchy are identified with the Stokes multipliers and their generalization [2] in the vacuum sector. Thus the result provides a unified view of Stokes multipliers and spectral determinant.

The spectral determinant for the wider class of potentials $x^{2 M}+\ell(\ell+1) / x^{2}$ with $M$ general can be treated within a framework using Baxter's $Q$ - operator and the quantum Wronskian relation 24].

We extend a part of studies in [2] to the higher order differential equation case,

$$
\partial^{n+1} y+(-1)^{n}\left(x^{m}+\lambda^{n+1}\right) y=0,
$$

with $m$ integer.

The $\lambda=0$ case is essentially equivalent to the Turrittin equation of which solutions are calculated in terms of Meijier $G$ - functions. There have been several results on this case 25, 26, 27.

We consider the Stokes multipliers associated with eq.(11). They satisfy a set of functional relations in the complex $\lambda$ plane. We will show that nontrivial solutions to relations are expressible by the quantum Jacobi-Trudi formula which appears in fusion transfer matrices related to $U_{q}\left(A_{n}^{(1)}\right)$ [28, 29].

We note the very recent result in [30] where the eigenvalue problem of eq.(1) is argued for $n=2$ case with $x \in[0,+\infty]$ though a related but a different method .

\section{Asymptotic Expansion and Stokes Coefficients}

We refer 31] to readers for the background on the subject.

Let us first discuss the asymptotic behavior of a slightly generalized differential equation,

$$
\begin{aligned}
& \partial^{n+1} y+(-1)^{n} P(x) y=0 \\
& P(x)=\sum_{j=0}^{m} a_{j} x^{m-j}
\end{aligned}
$$


where $a_{j}$ are complex numbers and $a_{0}=1 . P(x)$ will be referred to as the potential term in analogy with Schrödinger equations. I The factor $(-1)^{n}$ is not essential. It can be adsorbed into re-definition of the angle of $x$ and $a_{j \geq 1}$. For later convenience, we include this factor throughout this report.

Now that $x=\infty$ is an irregular singular point of the equation, analytic properties of solutions depend on sectors in the complex $x$ plane. Let $\mathcal{S}_{k}$ be a sector in the plane satisfying

$$
|\arg x-k \theta| \leq \frac{\theta}{2}
$$

for $x \in \mathcal{S}_{k}$, where $\theta=\frac{2 \pi}{m+n+1}$. We then study the asymptotic behavior of a subdominant solution in $\mathcal{S}_{0}$. Following [33, 34], we define $b_{h}(h=1,2, \cdots)$ by the relation,

$$
\left(1+\sum_{k=1}^{m} a_{k} x^{-k}\right)^{1 /(n+1)}=1+\sum_{h=1}^{\infty} b_{h} x^{-h} .
$$

A key function $E(x, \mathbf{a})$ is determined by $b_{h}$,

$$
\begin{aligned}
E(x, \mathbf{a}) & :=\int\left(1+\sum_{h=1}^{h_{m}} b_{h} x^{-h}\right) x^{m /(n+1)} d x \\
& =\frac{n+1}{m+n+1} x^{(m+n+1) /(n+1)}+\sum_{h=1}^{h_{m}} \frac{b_{h}}{\frac{m}{n+1}-h+1} x^{m /(n+1)+1-h}
\end{aligned}
$$

where $h_{m}=N$ for $m=N(n+1)-j,(j=1, \cdots, n)$. Here a stands for $\left(a_{1}, a_{2}, \cdots, a_{m}\right)$

In addition, we introduce an exponent $\nu_{m}$ by

$$
\nu_{m}=\begin{aligned}
\frac{n m}{2}, & \text { for } m \neq 0 \bmod n+1 \\
\frac{n m}{2}+(n+1) b_{h_{m}+1}, & \text { for } m=0 \bmod n+1
\end{aligned}
$$

Then we have a theorem,

Theorem 1 In $\mathcal{S}_{0}$, there exists a subdominant solution $y(x, \mathbf{a})$ to eq. (因) which admits the following asymptotics,

$$
\begin{aligned}
y(x, \mathbf{a}) & \sim x^{-\nu_{m} /(n+1)} e^{-E(x, \mathbf{a})}, \\
\partial^{j} y(x, \mathbf{a}) & \sim x^{\left(j m-\nu_{m}\right) /(n+1)} e^{-E(x, \mathbf{a})}, \quad j \geq 1 .
\end{aligned}
$$

The range of the existence of the asymptotic expansion (四) (forgetting the subdominance) actually extends over $\mathcal{S}_{0}$. The precise determination of the range, however, poses a nontrivial problem even for $n=1$ case. See elaborate discussions in sections 9 and 10

\footnotetext{
${ }^{1}$ In the case of singularity at infinity of rank 1 , i.e., $P(x)=p_{0}+p_{1} / x+\cdots$ in the above, asymptotic solutions and numerical algorithms are discussed in [32 for a wider class of higher order differential equations.
} 
of [34]. The straightforward extension of the argument may lead to the conclusion that $y(x, \mathbf{a})$ admits the asymptotic expansion (4) in an open sector,

$$
\arg x<\frac{n+2}{m+n+1} \pi
$$

See the appendix.

The intriguing feature in the differential equation (2) is the following symmetry in rotating the $x$ - plane.

Theorem 2 Denote the above solution by $y(x, \mathbf{a})$. Then

$$
y_{k}(x, \mathbf{a}):=y\left(x q^{-k}, G^{(k)}(\mathbf{a})\right) q^{n k / 2}
$$

is also a solution to eq. (国).

The parameter $q$ signifies $\exp (i \theta)=\exp \left(i \frac{2 \pi}{m+n+1}\right)$. The operation $G^{(k)}(\mathbf{a})$ is defined by $G^{(k)}(\mathbf{a})=G^{(1)}\left(G^{(k-1)}(\mathbf{a})\right), k \geq 2$ and $G^{(1)}(\mathbf{a})=\left(a_{1} / q, a_{2} / q^{2}, \cdots a_{m} / q^{m}\right)$.

A fundamental system of solutions (FSS) in $\mathcal{S}_{k}$ is formed by $\left(y_{k}, y_{k+1}, \cdots, y_{k+n}\right)$. This is shown for the Turrittin equation in [27]. For general, it is not simple to show this directly. We use the range of the validity of asymptotic expansion in eq.(司) and justify the linear independence as follows.

We introduce a $(n+1) \times(n+1)$ matrix $\Phi_{k}(x)$ and the Wronskian $W_{k}:=\operatorname{det} \Phi_{k}(x)$

$$
\Phi_{k}(x):=\left(\begin{array}{cccc}
y_{k}, & y_{k+1}, & \cdots, & y_{k+n} \\
\partial y_{k}, & \partial y_{k+1}, & \cdots, & \partial y_{k+n} \\
\vdots & & & \vdots \\
\partial^{n} y_{k}, & \partial^{n} y_{k+1}, & \cdots, & \partial^{n} y_{k+n}
\end{array}\right)
$$

Then eq.(5) implies that $y_{k+j}, j=0, \cdots, n$ has the asymptotic expansion (6) in the closed sector $\mathcal{S}_{k+(n+1) / 2} \cup \mathcal{S}_{k+(n-1) / 2}$. As $W_{k}$ is constant in $x$, one then verifies the linear independence of these solutions by using the asymptotic expansions (田) and (6) in $\mathcal{S}_{k+(n+1) / 2} \cup \mathcal{S}_{k+(n-1) / 2}$. See discussions in [34] for $n=1$ and [30] for $n=2$

We stress that the argument below depends on the existence of $\Phi_{k}(x)$ as FSS, rather than (5).

A matrix of Stokes multipliers $S_{k}^{(1)}$ connects FFS of $\mathcal{S}_{k}$ and $\mathcal{S}_{k+1}$

$$
\Phi_{k}(x)=\Phi_{k+1}(x) S_{k}^{(1)} .
$$

The linear independence of solutions fixes $S_{k}$ in the following form,

$$
S_{k}^{(1)}=\left(\begin{array}{cccccc}
\tau_{k}^{(1)}, & 1, & 0, & 0, & \cdots, & 0 \\
\tau_{k}^{(2)}, & 0, & 1, & 0, & \cdots & 0 \\
\vdots & & & & & \vdots \\
\tau_{k}^{(n)}, & 0, & 0, & 0, & \cdots & 1 \\
\tau_{k}^{(n+1)}, & 0, & 0, & 0, & \cdots & 0
\end{array}\right)
$$


By Cramer's formula, one represents $\tau_{k}^{(j)}$ as

$$
\tau_{k}^{(j)}=\frac{1}{W_{k}} \operatorname{det}\left(\begin{array}{cccccc}
y_{k+1}, & y_{k+2}, & \cdots, & y_{k}, & \cdots, & y_{k+n+1} \\
\vdots & & & & & \vdots \\
\partial^{n} y_{k+1}, & \partial^{n} y_{k+2}, & \cdots, & \partial^{n} y_{k}, & \cdots, & \partial^{n} y_{k+n+1}
\end{array}\right)
$$

The column vector, $\left(y_{k}, \partial y_{k}, \cdots, \partial^{n} y_{k}\right)$ is inserted in the $j$-th column in the matrix of the denominator. Especially,

$$
\tau_{k}^{(n+1)}=(-1)^{n} W_{k+1} / W_{k} .
$$

Then we restrict ourselves to the case of our interest (i1). We find that $b_{h_{m}+1}=0$ and thus $\nu_{m}=n m / 2$ for any $m$. Under the operation of $G^{(1)}, G^{(1)}\left(a_{m}\right)=a_{m} q^{-m}=a_{m} q^{n+1}$. As $a_{m}=\lambda^{n+1}$, this means $G^{(1)}(\lambda)=\lambda q$. Thus a function in the $k-$ th sector has an argument $\lambda q^{k}$.

\section{Recursion relations}

By the commensurability of the cone angle of $\mathcal{S}_{k}$ to $2 \pi$, the product of successive $m+n+1$ Stokes matrices must be a unit matrix [33] modulo sign factor due to the normalization in (6).

$$
S_{m+n}^{(1)} S_{m+n-1}^{(1)} \cdots S_{0}^{(1)}=(-1)^{n} I .
$$

Regarding as a function of $\lambda, S_{k}^{(1)}=S^{(1)}\left(\lambda q^{k}\right)$. Thus eq. (12) reads,

$$
S^{(1)}\left(\lambda q^{m+n}\right) S^{(1)}\left(\lambda q^{m+n-1}\right) \cdots S^{(1)}(\lambda)=(-1)^{n} I .
$$

The same relation can be recapitulated in terms of a generalized Stokes matrix $S_{k}^{(\ell)}$ connecting $\Phi_{k}$ and $\Phi_{k+\ell}$ [2, 31],

$$
\Phi_{k}=\Phi_{k+\ell} S_{k}^{(\ell)}
$$

Similar to the above, $S_{k}^{(\ell)}=S^{(\ell)}\left(\lambda q^{k}\right)$ as a function of $\lambda$.

Eq. (13) is obviously rewritten as,

$$
S^{(m+n+1)}(\lambda)=(-1)^{n} I .
$$

By the above definition, we have two equivalent recursion relations

$$
\begin{aligned}
S^{(\ell+1)}(\lambda) & =S^{(1)}\left(\lambda q^{\ell}\right) S^{(\ell)}(\lambda) \\
& =S^{(\ell)}(\lambda q) S^{(1)}(\lambda) .
\end{aligned}
$$

We denote the $(i, j)$ component of $S^{(\ell)}(\lambda)$ by $S_{i, j}^{(\ell)}(\lambda)$.

The main problems in the present report are 
(1) the expression of $S_{i, j}^{(\ell)}(\lambda)$ in terms of $\tau^{(a)}(\lambda) \mathrm{s}$

(2) restrictions imposed on $\tau^{(a)}(\lambda)$ s by functional relations among them.

The first recursion relation (16) imposes the relations,

$$
S_{k, j}^{(\ell+1)}(\lambda)=\tau^{(k)}\left(q^{\ell} \lambda\right) S_{1, j}^{(\ell)}(\lambda)+S_{k+1, j}^{(\ell)}(\lambda) \quad(1 \leq k \leq n+1)
$$

where we formally put $S_{n+2, j}^{(\ell)}(\lambda)=0$

The second recursion relation (17) yields,

$$
\begin{aligned}
S_{k, 1}^{(\ell+1)}(\lambda)= & \tau^{(1)}(\lambda) S_{k, 1}^{(\ell)}(\lambda q)+\tau^{(2)}(\lambda) S_{k, 2}^{(\ell)}(\lambda q)+ \\
& \cdots+\tau^{(n+1)}(\lambda) S_{k, n+1}^{(\ell)}(\lambda q)
\end{aligned}
$$

and

$$
S_{i, j+1}^{(\ell+1)}(\lambda)=S_{i, j}^{(\ell)}(q \lambda) \quad(1 \leq i \leq n+1,1 \leq j \leq n) .
$$

The latter equation leads to

$$
S_{i, j+1}^{(\ell)}(\lambda)=S_{i, 1}^{(\ell-j)}\left(q^{j} \lambda\right) \quad \ell \geq j .
$$

For $\ell<j$,

$$
S_{i, j}^{(\ell+1)}(\lambda)=S_{i, j-\ell}^{(1)}\left(q^{\ell} \lambda\right)
$$

and the rhs is given directly in terms of $\tau^{(a)}$. Thus we regard only $S_{k, 1}^{(\ell)}$ as nontrivial elements.

The eq. (19) can be rewritten such that it contains only $S_{k, 1}^{(\ell)}$ in the lhs. Let us write explicitly for $k=1$,

$$
\begin{aligned}
S_{1,1}^{(\ell+1)}(\lambda)= & \tau^{(1)}(\lambda) S_{1,1}^{(\ell)}(\lambda q)+\tau^{(2)}(\lambda) S_{1,1}^{(\ell-1)}\left(\lambda q^{2}\right)+ \\
& \cdots+\tau^{(n+1)}(\lambda) S_{1,1}^{(\ell-n)}\left(\lambda q^{n+1}\right) .
\end{aligned}
$$

We remark properties of $S_{1,1}^{(\ell)}(\lambda)$ from linear independency of FSS. The $(1,1)$ component of eq.(14) for $\ell=m+j, 1 \leq j \leq n$ reads,

$$
y_{k}=S_{1,1}^{(m+j)} y_{k+m+j}+\cdots .
$$

$\Phi_{k+m+j}$ consists of $\left(y_{k+m+j}, y_{k+m+j+1}, \cdots, y_{k+m+n+j}\right)$. As $y_{k+m+n+j}=(-1)^{n} y_{k+j-1}$, it contains $(-1)^{n} y_{k}$. Thus the linear independency of solutions concludes at least

$$
S_{1,1}^{(m+j)}=0, \quad 1 \leq j \leq n .
$$

We also note a trivial relation, $S_{1,1}^{(m+n+1)}=(-1)^{n}$.

In the next section, we will show that a set of solutions to the above recursion relations can be neatly represented by the quantum Jacobi-Trudi formula. 


\section{Quantum Jacobi-Trudi formula in Stokes multipli- ers}

The recursion relation (23) can be successively solved for given initial conditions $\tau^{(1)}(\lambda), \cdots, \tau^{(n)}(\lambda)$ (forward-propagation). Conversely, $\tau^{(a)}(\lambda)$ is expressible by these solutions (back-propagation).

To represent these, we employ notations useful in later discussions,

$$
\begin{aligned}
& T_{\ell}^{(1)}(\lambda)=S_{1,1}^{(\ell)}\left(\lambda q^{-(\ell-1) / 2}\right) \\
& T_{1}^{(a)}(\lambda)=(-1)^{a+1} \tau^{(a)}\left(\lambda q^{-(a-1) / 2}\right) \quad\left(=(-1)^{a+1} S_{a, 1}^{(1)}\left(\lambda q^{-(a-1) / 2}\right)\right) .
\end{aligned}
$$

We first state the result for the forward-propagation problem,

$$
T_{\ell+1}^{(1)}(\lambda)=\operatorname{det}\left(T_{1}^{(1-i+j)}\left(\lambda q^{(\ell+2-i-j) / 2}\right)\right)_{1 \leq, i, j \leq \ell+1} .
$$

The rhs includes the formal extension of $T_{1}^{(a)}(\lambda)$, and we understand $T_{1}^{(a)}(\lambda)=0$ for $a \geq n+2$ or $a<0$ and $T_{1}^{(0)}(\lambda)=1$.

The proof is easy by the induction on $\ell$. The case $\ell=0$ is trivial by definition. Assume that eq.(25) is valid up to $\ell$. Expanding the determinant with respect to the last column, we have

$$
\begin{aligned}
T_{\ell+1}^{(1)}(\lambda)= & T_{1}^{(1)}\left(\lambda q^{-\ell / 2}\right) T_{\ell}^{(1)}\left(\lambda q^{1 / 2}\right)-T_{1}^{(2)}\left(\lambda q^{-(\ell-1) / 2}\right) T_{\ell-1}^{(1)}(\lambda q) \\
& +\cdots+(-1)^{a+1} T_{1}^{(a)}\left(\lambda q^{-(\ell+1-a) / 2}\right) T_{\ell+1-a}^{(1)}\left(\lambda q^{a / 2}\right)+\cdots
\end{aligned}
$$

Here we set $T_{\ell}^{(1)}(\lambda)=0, \ell<0$ and $T_{0}^{(1)}(\lambda)=1$.

By eqs. (25), (26) and from the assumption of the induction, the lhs is equal to that in eq.(23) after $\lambda \rightarrow \lambda q^{-\ell / 2}$. Then the validity of eq.(25) carries over into $\ell+1$, which completes the proof.

Next consider the back-propagation problem. We regard the coupled equations (23) for $\ell=0,1,2, \cdots, n$, as a set of linear equations for $\tau^{(a)}(\lambda)\left(\right.$ or $\left.T_{1}^{(a)}(\lambda)\right)$. The Cramer's theorem is again applicable. Taking account of the fact that the determinant of the coefficient matrix is unity, we have,

$$
T_{1}^{(a)}(\lambda)=\operatorname{det}\left(T_{1-i+j}^{(1)}\left(\lambda q^{(i+j-a-1) / 2}\right)\right)_{1 \leq, i, j \leq a} .
$$

It is natural to define $T_{1}^{(0)}(\lambda)=1$ from this expression.

We notice the similarity of eqs. (27) and (29) to the Jacobi-Trudi formula for Schur functions [35]. It states that any complex Schur function associated to a skew Young diagram can be represented in terms of a determinant of a matrix whose elements are given by elementary Schur functions.

We find a quite parallel result in the present problem. Thanks to the argument around eqs.(21) and (22), only $S_{k, 1}^{(\ell)}(\lambda)$ is of our interest. This turns out to have a compact expression in a similar manner to the Jacobi-Trudi formula, which we call the quantum Jacobi-Trudi formula. 
To state this, we prepare some notations. By $\mu$, we mean a Young diagram $\left(\mu_{1}, \mu_{2}, \cdots\right)$ and by $\mu^{\prime}$, its transpose. Consider a skew Young table of the shape $\mu / \eta=\left(\mu_{1}-\eta_{1}, \mu_{2}-\right.$ $\left.\eta_{2}, \cdots\right)$ such that $\mu_{i} \geq \eta_{i}$. We define a quantity associated to $\mu / \eta$,

$$
T_{\mu / \eta}(\lambda):=\operatorname{det}_{1 \leq j, k \leq \mu_{1}^{\prime}}\left(T_{\mu_{j}-\eta_{k}-j+k}^{(1)}\left(\lambda q^{-\left(\mu_{1}^{\prime}-\mu_{1}+\mu_{j}+\eta_{k}-j-k+1\right) / 2}\right)\right) .
$$

It contains eq.(29) as a special case $\mu=(1,1, \cdots, 1)$ and $\eta=\phi$.

A generalization of eq. (27) also exists. To show this, consider two matrices $H$ and $E$ with entries

$$
\begin{aligned}
H_{i, j} & :=(-1)^{i-j} T_{i-j}^{(1)}\left(\lambda q^{-(i+j) / 2}\right), \\
E_{i, j} & :=T_{1}^{(i-j)}\left(\lambda q^{-(i+j) / 2}\right) .
\end{aligned}
$$

Clearly, $\operatorname{det} H=\operatorname{det} E=1$ as they are lower triangular with all diagonal elements unity. The expansion of eq.(27) with respect to the 1st column leads to

$$
\begin{aligned}
0= & -T_{\ell+1}^{(1)}(\lambda)+T_{1}^{(1)}\left(\lambda q^{\ell / 2}\right) T_{\ell}^{(1)}\left(\lambda q^{-1 / 2}\right)-T_{1}^{(2)}\left(\lambda q^{(\ell-1) / 2}\right) T_{\ell-1}^{(1)}\left(\lambda q^{-1}\right) \\
& +\cdots+(-1)^{a+1} T_{1}^{(a)}\left(\lambda q^{(\ell+1-a) / 2}\right) T_{\ell+1-a}^{(1)}\left(\lambda q^{-a / 2}\right)+\cdots
\end{aligned}
$$

which is equivalent to

$$
\delta_{i, j}=\sum_{k}(-1)^{i-k} T_{i-k}^{(1)}\left(\lambda q^{-(i+k) / 2}\right) T_{1}^{(k-j)}\left(\lambda q^{-(k+j) / 2}\right) .
$$

Hence $E$ and $H$ are inverse each other.

These two facts are sufficient for the second representation of $T_{\mu / \eta}$ [28, 35, 39],

$$
T_{\mu / \eta}(\lambda)=\operatorname{det}_{1 \leq j, k \leq \mu_{1}}\left(T_{1}^{\left(\mu_{j}^{\prime}-\eta_{k}^{\prime}-j+k\right)}\left(\lambda q^{-\left(\mu_{1}^{\prime}-\mu_{1}-\mu_{j}^{\prime}-\eta_{k}^{\prime}+j+k-1\right) / 2}\right)\right) .
$$

Eqs. (30) and (31) coincide with the Jacobi-Trudi formula by dropping the $\lambda$ dependencies.

We have the following statement on nontrivial Stokes multipliers.

Theorem 3 The solution to the recursion relation (18) for $j=1$ is given by

$$
S_{k, 1}^{(\ell)}(\lambda)=(-1)^{k+1} T_{\mu}\left(q^{(\ell+k-2) / 2} \lambda\right),
$$

where $\mu$ is a Young diagram of the hook shape $\mu=(\ell, 1, \cdots, 1)$ with height $k$.

The proof is as follows. In terms of $T$, we need to show

$$
T_{\mu^{\prime}}(\lambda)+T_{\mu^{\prime \prime}}(\lambda)=T_{1}^{(k)}\left(\lambda q^{\ell / 2}\right) T_{\ell}^{(1)}\left(\lambda q^{-k / 2}\right),
$$

where $\mu^{\prime}\left(\mu^{\prime \prime}\right)$ is also the hook Young diagram $\mu^{\prime}=(\ell+1,1, \cdots, 1),(\mu "=(\ell, 1, \cdots, 1))$ with height $k(k+1)$. Then the equality is verified by expanding the determinant associated to $T_{\mu "}(\lambda)$ with respect to the first row. 
It remains to show the consistency of eq. (32) with eqs. (18) $(k=n+1$ and $j=1)$ and (15).

First, consider $k=n+1$ and $j=1$ of eq.(18). By the recursion argument in the above, the formal extension $S_{n+2,1}^{(\ell)}(\lambda)$, defined by the rhs of eq.(32), must be zero. This is directly seen from the second expression (31), as $T_{1}^{(a)}(\lambda)=0,(a \geq n+2)$.

Next we deal with eq.(15). To be precise we will show

$$
S_{i, j}^{(m+n+1)}(\lambda)=T_{m+n+1}^{(1)}\left(\lambda q^{(m+n) / 2}\right) \delta_{i, j} \quad\left(=(-1)^{n} \delta_{i, j}\right.
$$

With slight re-definition of index $j$ and the application of the relation (21), this is converted into an equivalent form,

$$
S_{i, 1}^{(m+j+1)}(\lambda)=T_{m+n+1}^{(1)}\left(\lambda q^{(m+n) / 2}\right) \delta_{i+j, n+1} \quad(0 \leq j \leq n) .
$$

We shall divide argument into two cases, $j \neq 0$ and $j=0$.

For $j=0$, we apply eq.(32) to $S_{i, 1}^{(m+1)}(\lambda)$. Then we find from the formula (30) that the all elements of the first row are zero unless $i=n+1$. This comes from eq.(24), $T_{m+j^{\prime}}(\lambda)=0$ for $1 \leq j^{\prime} \leq n$. For $i=n+1$, We expand the determinant with respect to the last column. From the normalization in eq.(32) and $T_{0}^{(1)}(\lambda)=1$, it follows $S_{n+1,1}^{(m+1)}(\lambda)=$ $T_{m+n+1}^{(1)}\left(\lambda q^{(m+n) / 2}\right)$, which is $(-1)^{n}$ by definition.

A remark is in order. The second form (31) leads to $S_{n+1,1}^{(m+1)}(\lambda)=$ $\left.(-1)^{n} T_{m}^{(1)}\left(\lambda q^{(m-1) / 2}\right) T_{1}^{(n+1)}\left(\lambda q^{3 m+n / 2}\right)\right)$. Then

$$
T_{m}^{(1)}\left(\lambda q^{-(n+1) / 2}\right) T_{1}^{(n+1)}\left(\lambda q^{m / 2}\right)=(-1)^{n} T_{m+n+1}^{(1)}(\lambda)=1
$$

must hold. Neither $T_{m}^{(1)}(\lambda)$ or $T_{1}^{(n+1)}(\lambda)$ has poles. Thus eq.(35) asserts that they are also nonzero everywhere. Consequently they are constant. We drop their $\lambda$ dependencies for the time being. By taking the determinants of both sides of (matrix form of) eq.(33), one obtains $\left(T_{1}^{(n+1)}\right)^{m+n+1}=\left(T_{m+n+1}^{(1)}\right)^{n+1}$. Being combined with eq.(35), this yields, $\left(T_{1}^{(n+1)}\right)^{m}=\left(T_{m}^{(1)}\right)^{n+1}$. We conclude both $T_{1}^{(n+1)}$ and $T_{m}^{(1)}$ are root of unity. The former can be derived directly once if we assume (5) and use (11). One only has to evaluate $W_{k}$ using asymptotic forms (4) and (6) in $\mathcal{S}_{k+(n+1) / 2} \cup \mathcal{S}_{k+(n-1) / 2}$. The simple manipulation yields $\tau_{k}^{(n+1)}=(-1)^{n}$, thus $T_{1}^{(n+1)}=1$.

For $j \neq 0$, the recursion (18) yields,

$$
\begin{aligned}
S_{i, 1}^{(m+j+1)}(\lambda) & =\tau^{(i)}\left(\lambda q^{m+j}\right) S_{1,1}^{(m+j)}(\lambda)+S_{i+1,1}^{(m+j)}(\lambda) \\
& =(-1)^{i} T^{(i)}\left(\lambda q^{m+j+(i-1) / 2}\right) T_{m+j}^{(1)}\left(\lambda q^{(m+j-1) / 2}\right)+S_{i+1,1}^{(m+j)}(\lambda)
\end{aligned}
$$

In the present case, the first term in eq.(36) is vanishing due to eq.(24). Thus

$$
S_{i, 1}^{(m+j+1)}(\lambda)=S_{i+1,1}^{(m+j)}(\lambda)
$$


We then try to prove eq.(34) by induction on the upper index, starting from the result for $S_{i, 1}^{(m+1)}(\lambda)$ as the "initial condition". In each induction step, however, $i=n+1$ component is indeterminate from eq.(37). We then use eq.(31) and find $S_{n+1,1}^{(\ell)}(\lambda)=$ $(-1)^{\ell+1} T_{\ell-1}^{(1)}\left(\lambda q^{\omega(\ell)}\right) T_{1}^{(n+1)}\left(\lambda q^{\omega^{\prime}(\ell)}\right)$ where $\omega(\ell)$ and $\omega^{\prime}(\ell)$ are some shifts irrelevant in the present argument. This supplies the missing pieces. As $\ell \geq m+2$ for the case under consideration, these are null. We then immediately check that eq.(34) holds.

Thereby, we prove that nontrivial Stokes multipliers are explicitly given via formula (32). The fundamental quantities $\tau^{(a)} \mathrm{s}$ are not all independent. Constraints (24) impose complex algebraic equations among them.

We note useful relations among $T$ s, the $T$ - system. Let $T_{\ell}^{(a)}(\lambda)=T_{\mu}(\lambda)$ for $\mu$ being a rectangle of height $a$ and width $\ell$. By applying the Plücker relation to eq.(30) or eq.(31), one finds,

$$
T_{\ell}^{(a)}\left(\lambda q^{1 / 2}\right) T_{\ell}^{(a)}\left(\lambda q^{-1 / 2}\right)=T_{\ell+1}^{(a)}(\lambda) T_{\ell-1}^{(a)}(\lambda)+T_{\ell}^{(a+1)}(\lambda) T_{\ell}^{(a-1)}(\lambda), \quad a=1, \cdots, n .
$$

The boundary conditions are $T_{\ell<0}^{(1)}(\lambda)=T_{m+1 \leq \ell \leq m+n}^{(1)}(\lambda)=T_{1}^{(a<0)}(\lambda)=T_{1}^{(a>n+1)}(\lambda)=0$ and $T_{\ell}^{(a)}(\lambda)=1$ if $a=0$ or $\ell=0$. The last relation comes from the null dimensionality of the matrix in eqs. (30) and (31). The initial condition $T_{\ell<0}^{(1)}(\lambda)=0$ leads $T_{\ell<0}^{(a)}(\lambda)=0,(1 \leq$ $a \leq n)$. Similarly we have $T_{m+j}^{(a)}(\lambda)=0(1 \leq a, j \leq n)$. Thus the $T$ - system constitutes finite number of relations with finite number of $T_{\ell}^{(a)}(\lambda)$.

Remark that the case with $n=1, m=3$ and with multi-parameters has been found in [34 by direct calculation of recursion relations. The one parameter result has been later extended to arbitrary $m$ by similar argument employed here [2].

Summarizing this section, we have found determinant representations of Stokes multipliers. We conveniently introduce a subset of huge hierarchy, $T_{\ell}^{(a)}(\lambda)$ including (the most fundamental) Stokes multipliers $\tau^{(a)}(\lambda)$. Then the functional relations exist among them which, in turns, impose some restrictions on $\tau^{(a)}(\lambda)$.

We will discuss above results in view of solvable models in the next section. Especially we will give some follow-up of the last sentence in the previous paragraph.

\section{Functional relations in solvable models and Ther- modynamic Bethe Ansatz}

Hereafter we assume $n$ odd and $m=M(n+1)$. It is then convenient to rotate the $\lambda$ for the differential equation (11) by $\frac{\pi}{2}$. We will use the same notation for the resultant $\lambda$.

In the first part of this section, we remind of some concrete results in solvable models. The second part is devoted to a rather speculative discussion of the possibility of the application of Thermodynamic Bethe ansatz to the evaluation of Stokes multipliers.

The commuting transfer matrices play a fundamental role in studies of solvable lattice models and field theories [11, 12, 13]. The members in a commuting family share the same physical space ( quantum space) and are parameterized by the (multiplicative) spectral 
parameter $\lambda$. They are labeled by the auxiliary space of which trace must be taken. These auxiliary spaces are identified with irreducible modules of Yangian or quantum affine Lie algebra [37, 38]. For $U_{q}\left(A_{n}^{(1)}\right)$, there exists a irreducible module $W_{m}^{(a)}(\lambda),(a \leq n)$ which is isomorphic to $m V_{\Lambda_{a}}$ as a classical module. Naturally, we associate a Young diagram of $a \times m$ to this module, and write the corresponding transfer matrix $\mathcal{T}_{m}^{(a)}(\lambda)$. Since they are commutative, we consider transfer matrices on common eigenstates. Thus we sometimes do not distinguish operators from their eigenvalues.

In the language of solvable lattice models, $\mathcal{T}_{m}^{(a)}(\lambda)$ is a transfer matrix of a model obtained by the fusion procedure. Starting from a "fundamental" model acting on $V_{\Lambda_{1}} \times$ $V_{\Lambda_{1}}$, we can recursively derive fusion models. Their auxiliary spaces are constructed from $V_{\Lambda_{1}} \times \cdots \times V_{\Lambda_{1}}$ by applying appropriate projectors. We utilize singular points of $R$ - matrix for construction of these projectors.

The fusion procedure is not restricted to the rectangle type. Generally, there exists a module $W_{\mu / \eta}(\lambda)$, or a fusion model, parameterized by a skew Young diagram $\mu / \eta$. We write the corresponding transfer matrix as $\mathcal{T}_{\mu / \eta}(\lambda)$.

The short exact sequence of irreducible modules examined in [36, 28, 29] leads to the relation which we have seen in eq.(30),

$$
\begin{aligned}
\mathcal{T}_{\mu / \eta}(\lambda) & =\operatorname{det}_{1 \leq j, k \leq \mu_{1}}\left(\mathcal{T}_{1}^{\left(\mu_{j}^{\prime}-\eta_{k}^{\prime}-j+k\right)}\left(\lambda q^{-\left(\mu_{1}^{\prime}-\mu_{1}-\mu_{j}^{\prime}-\eta_{k}^{\prime}+j+k-1\right) / 2}\right)\right) \\
& =\operatorname{det}_{1 \leq j, k \leq \mu_{1}^{\prime}}\left(\mathcal{T}_{\mu_{j}-\eta_{k}-j+k}^{(1)}\left(\lambda q^{-\left(\mu_{1}^{\prime}-\mu_{1}+\mu_{j}+\eta_{k}-j-k+1\right) / 2}\right)\right)
\end{aligned}
$$

where $\mathcal{T}_{m<0}^{(1)}(\lambda)=\mathcal{T}_{1}^{(a<0)}(\lambda)=0$.

This assures the $T$ - system (38) among $\mathcal{T}$ [29]. The quantum Jacobi Trudi formula plays a role in several problems in solvable models [39, 40, 41]

The conditions (24) also hold. When $q=\exp \left(i \frac{2 \pi}{m+n+1}\right)$, the truncation of the space happens due to quantum group symmetry. In view of solvable lattice models, it corresponds to the situation that some projectors are vanishing and fusion paths in local variables are lost. Consequently, no local variables can be adjacent and transfer matrices vanish 28],

$$
\mathcal{T}_{m+1}^{(a)}(\lambda)=\mathcal{T}_{m+2}^{(a)}(\lambda)=\cdots=\mathcal{T}_{m+n}^{(a)}(\lambda)=0
$$

The normalizations of $\mathcal{T}_{0}^{(a)}(\lambda), \mathcal{T}_{\ell}^{(0)}(\lambda)$ and $\mathcal{T}_{\ell}^{(n+1)}(\lambda)$ depend on choice of quantum space. We have not yet found the description of the quantum space yielding $\mathcal{T}_{0}^{(a)}(\lambda)=$ $\mathcal{T}_{\ell}^{(0)}=\mathcal{T}_{\ell}^{(n+1)}=1$, except for $n=1$. We assume the existence of it for general $n$.

Then, on the corresponding space, the fusion transfer matrices share same functional relations with Stokes multipliers.

Below we use the same symbol $T_{\ell}^{(a)}(\lambda)$ for these two cases.

Thanks to the (quantum group) reduction,the $T$ - system closes within a finite set of unknowns.

The solution to functional relations, however, is not unique. Additional information on the analyticity of $T_{\ell}^{(a)}(\lambda)$ in the complex $\lambda$ are required for the uniqueness. 
We start from the lattice model. Then we take the scaling limit (or the field theoretical limit) which has been discussed in several literatures. In the present context, we refer to [42]. Note that our transfer matrices are not mere sums of products of Boltzmann weights but are imposed overall renormalizations due to $\mathcal{T}_{0}^{(a)}(\lambda)=\mathcal{T}_{\ell}^{(0)}=\mathcal{T}_{\ell}^{(n+1)}=1$.

Let us define an "additive" spectral parameter $u$ by $\lambda=\exp \left(\frac{\pi u}{(m+n+1)}\right)$. We also introduce

$$
Y_{\ell}^{(a)}(u):=\frac{T_{\ell+1}^{(a)}(\lambda(u)) T_{\ell-1}^{(a)}(\lambda(u))}{T_{\ell}^{(a+1)}(\lambda(u)) T_{\ell}^{(a-1)}(\lambda(u))} \quad(1 \leq a \leq n, 1 \leq \ell \leq m) .
$$

Then eq.(38) reads

$$
Y_{\ell}^{(a)}(u+i) Y_{\ell}^{(a)}(u-i)=\frac{\left(1+Y_{\ell+1}^{(a)}(u)\right)\left(1+Y_{\ell-1}^{(a)}(u)\right)}{\left(1+\left(Y_{\ell}^{(a+1)}(u)\right)^{-1}\right)\left(1+\left(Y_{\ell}^{(a-1)}(u)\right)^{-1}\right)},
$$

where $\left(Y_{\ell}^{(0)}(u)\right)^{-1}=Y_{m}^{(a)}(u)=0$ by definition.

Remember that that Boltzmann weights are regular functions of $u$. Then, apart from some renormalization factors mentioned above, $T_{\ell}^{(a)}(\lambda(u))$ must not be singular in the strip $\Im u \in[-1,1]$ but may possess zeros in general.

Suppose that $T_{\ell}^{(a)}(\lambda(u))$ has finitely many distinct zeros in the strip $\left\{u_{\ell, k}^{(a)}\right\}$ which depend on eigenstates. They are assumed to be off the lines $\Im u= \pm 1$.

Then eq. (42) can be transformed into coupled nonlinear integral equations by the standard trick [17, 43, 12, 13, 44, 45].

The both sides of eq. (42) are analytic with finitely many distinct zeros and have constant asymptotics. Then, applying Cauchy's theorem, we have

$$
\begin{aligned}
& \log Y_{\ell}^{(a)}(u)=\log \mathcal{Z}_{\ell}^{(a)}(u) \\
& +\sum_{b=1}^{a} \sum_{r=1}^{m} \int_{-\infty}^{\infty} K\left(u-u^{\prime}\right) \log \frac{\left(1+Y_{\ell+1}^{(a)}\left(u^{\prime}\right)\right)\left(1+Y_{\ell-1}^{(a)}\left(u^{\prime}\right)\right)}{\left(1+\left(Y_{\ell}^{(a+1)}\left(u^{\prime}\right)\right)^{-1}\right)\left(1+\left(Y_{\ell}^{(a-1)}\left(u^{\prime}\right)\right)^{-1}\right)} d u^{\prime}+C_{\ell}^{(a)}(43)
\end{aligned}
$$

where $C_{\ell}^{(a)}$ is a "integral" constant fixed by comparing asymptotic values of both sides. $\mathcal{Z}_{\ell}^{(a)}(u)$ signifies,

$$
\begin{aligned}
& \mathcal{Z}_{\ell}^{(a)}(u)=\frac{Z_{\ell+1}^{(a)}(u) Z_{\ell-1}^{(a)}(u)}{Z_{\ell}^{(a+1)}(u) Z_{\ell}^{(a-1)}(u)} \\
& Z_{\ell}^{(a)}(u)=\prod_{j}\left(\tanh \frac{\pi}{4}\left(u-w_{\ell, j}^{(a)}\right)\right)^{\epsilon, j} \times \prod_{k} \tanh \frac{\pi}{4}\left(u-u_{\ell, k}^{(a)}\right) .
\end{aligned}
$$

and $Z_{\ell}^{(0)}(u)=Z_{\ell}^{(n+1)}(u)=Z_{0}^{(a)}(u)=1$.

Here $\left.\left\{w_{\ell, j}^{(a)}\right)\right\}$ is the joint set of zeros $\left(\epsilon_{\ell, j}=1\right)$ and singularities $\left(\epsilon_{\ell, j}=-1\right)$ of $T_{\ell}^{(a)}(u)$ in $\Im u \in[-1,1]$ due to the above normalization. They stem from common factors in Boltzmann weights, thus they are zeros or singularities of order $N(N=$ system size). Here we label them disregarding of their multiplicities. 
The kernel function is easily written in the Fourier transformed form. We define the Fourier transformation $\widehat{f}[k]$ of a function $f(u)$ by

$$
f(u)=\frac{1}{2 \pi} \int_{-\infty}^{\infty} \widehat{f}[k] e^{i k u} d u, \quad \widehat{f}[k]=\int_{-\infty}^{\infty} f(u) e^{i k u} d u,
$$

then

$$
\widehat{K}[k]=\frac{1}{2 \cosh k} .
$$

Eq. (43) might be represented by the following form, in analogy with thermodynamic Bethe ansatz equation in CFT,

$$
\begin{aligned}
& \widetilde{\log } \widetilde{Y_{\ell}^{(a)}}(u)=\sum_{r=1}^{m} \int_{-\infty}^{\infty} A^{\ell, r}\left(u-u^{\prime}\right) \log \mathcal{Z}_{r}^{(a)}\left(u^{\prime}\right) d u^{\prime} \\
& +\sum_{b=1}^{a} \sum_{r=1}^{m} \int_{-\infty}^{\infty} K_{a, b}^{\ell, r}\left(u-u^{\prime}\right) \log \left(1+\left(Y_{r}^{(b)}\left(u^{\prime}\right)\right)^{-1}\right) d u^{\prime}+D_{\ell}^{(a)}
\end{aligned}
$$

where $D_{\ell}^{(a)}$ is also some "integral" constant and $\widetilde{Y_{\ell}^{(a)}}(u)=Y_{\ell}^{(a)}(u) / Z_{\ell}^{(a)}(u)$.

The Fourier transformations of the kernel functions read,

$$
\begin{aligned}
\widehat{K}_{a, b}^{\ell, r}[k] & =\widehat{A}^{\ell, r}[k] \widehat{M}_{a, b}[k] \\
\widehat{A}^{\ell, r}[k] & =\frac{\sinh (\min (\ell, r) k) \sinh ((m-\max (\ell, r)) k)}{\sinh (m k) \sinh (k)} \\
\widehat{M}_{a, b}[k] & =2 \cosh k\left(\delta_{a, b}-\frac{I_{a, b}}{2 \cosh k}\right)
\end{aligned}
$$

and $I_{a, b}=1$ if they are on adjacent nodes of Dynkin diagram for $A_{n}$ and $I_{a, b}=0$ otherwise.

At this stage, one performs the field theoretical limit, system size $\rightarrow \infty$, lattice spacing $\rightarrow 0$, and sending elliptic nome $\rightarrow 0$ under some fine-tuning condition. Precisely speaking, this condition depends on the regime of the lattice model. We shall skip that detail here. We refer [42] for detail in the case of $A_{1}^{(1)}$.

Then it can be shown that $\left\{w_{\ell, j}^{(a)}\right\}$ accumulate in $\infty$ and reproduce "momentum" term in eq.(15) from the first term [1, 12, 13, 15, 42],

$$
\begin{aligned}
& \widetilde{\log } \widetilde{Y_{\ell}^{(a)}}(u)=M_{\ell}^{(a)} \exp \left(\frac{\pi}{m} u\right)+\sum_{r=1}^{m} \int_{-\infty}^{\infty} A^{\ell, r}\left(u-u^{\prime}\right) \log \mathcal{Z}_{r}^{(a)}\left(u^{\prime}\right) d u^{\prime} \\
& +\sum_{b=1}^{a} \sum_{r=1}^{m} \int_{-\infty}^{\infty} K_{a, b}^{\ell, r}\left(u-u^{\prime}\right) \log \left(1+\left(Y_{r}^{(b)}\left(u^{\prime}\right)\right)^{-1}\right) d u^{\prime}+D_{\ell}^{(a)} .
\end{aligned}
$$

$\mathcal{Z}_{r}^{(a)}\left(u^{\prime}\right)$ consists only of $\left\{u_{\ell, k}^{(a)}\right\}$ in (44). 
The rapidity $\theta$ in [1] is denoted by $\frac{\pi}{m} u$ here.

The resultant coupled integral equations can be recursively solved and yield a unique set of solutions to $Y_{\ell}^{(a)}(u)$, and then to $T_{\ell}^{(a)}(\lambda(u))$ for given $\left\{u_{\ell, k}^{(a)}\right\}$ and $M_{\ell}^{(a)}$. Thus one must adopt appropriate choice of these parameters to reproduce proper $T_{\ell}^{(a)}(\lambda(u))$ including $\tau^{(a)}(\lambda)$. Conversely, if two sets of functions satisfy the same relation (38) and share the same $\left\{u_{\ell, k}^{(a)}\right\}$ and $M_{\ell}^{(a)}$, then they are identical in the strip.

For solvable lattice models, the case studies on $U_{q}\left(A_{1,2,3}^{(1)}\right)$ indicate that $T_{\ell}^{(a)}(\lambda(u))$ does not possess zeros in the strip $\Im u \in[-1,1]$ when acting on the largest eigenvalue sector. That is, $\left\{u_{\ell, k}^{(a)}\right\}=\phi, a=1, \cdots n$ for all $p \leq m$.

This seems to be also the case with Stokes multipliers. Actually this is the case for $n=1$. That is, the proper Stokes multipliers are reproduced by $\left\{u_{\ell, k}^{(a)}\right\}=\phi, a=1, \cdots n$ for all $p \leq m$ but different choice of $M_{\ell}^{(a)}$ from lattice models or field theoretic model. Let me just present some argument for $n \geq 2$ case. Assume that the solution to eq. (35) is simple, $T_{1}^{(n+1)}=T_{m}^{(1)}=1$. As remarked above, the former equality is a direct consequence of (5) and (11). From our "boundary conditions", the $T$ - system is then invariant under the simultaneous transformations $(a, \ell) \rightarrow(n+1-a, m-\ell)$. This implies $T_{\ell}^{(a)}(\lambda)=T_{m-\ell}^{(n+1-a)}(\lambda)$. By $\pm \theta_{\ell}^{(a)}$, we mean the imaginary part of zero of $T_{\ell}^{(a)}(\lambda)$. Then a sum rule holds,

$$
\theta_{\ell}^{(a)}+\theta_{m-\ell}^{(n+1-a)}=m+n+1 .
$$

The simplest solution, $\theta_{\ell}^{(a)}=a+\ell$, is actually correct for $n=a=1$. Postulating this solution, we conclude $\left\{u_{\ell, k}^{(a)}\right\}=\phi, a=1, \cdots n$ for all $p \leq m$ also for Stokes multipliers.

We do not expect ill-behavior for small angle sector of $\lambda ; \tau^{(a)}(\lambda)$ might not be vanishing in the sector, or otherwise it harms linear dependency of FSS. The above choice of $\theta_{\ell}^{(a)}$ is consistent with this expectation.

Admitting these assumptions, we have a conjecture

Conjecture 1 The Stokes multipliers of eq.(1) are given by "vacuum expectation values" of fusion transfer matrices related to $U_{q}\left(A_{n}^{(1)}\right)$. Thus they are evaluated from eq. (妸) by forgetting the second term in the rhs .

The parameters $M_{\ell}^{(a)}, D_{\ell}^{(a)}$ must be tuned correctly as in [1] for $n=1$.

To be precise, overall normalization of $\lambda$ for the identification is not fixed by functional relations only. For $n=1$ the determination of this factor is quite straightforward [1, 2], due to the fact that only one subdominant solution exists in each $\mathcal{S}_{j}$. The spectral determinant is then identified with the fusion Stokes multiplier which connects the subdominant solution on the negative real axis to the dominant solution on the positive real axis. Then standard WKB arguments yield the identification of parameters, especially $M_{\ell}^{(1)}$ and the normalization of $\lambda$.

This can not be generalized straightforwardly for $n \geq 2$. Let me just comment on two fundamental problems. First, the meaning of eigenvalue problem is not necessary clear for higher order differential equations. The characterization of the eigenspace (it is the Hilbert 
space for $n=1$ ) is not obvious. Second, more technically, there are several subdominant solutions in each sector. We hope to clarify these issues in future publications.

Finally we comment on the $(1,1)$ component of eq.(8).

$$
y_{k}=\tau_{k}^{(1)} y_{k+1}+\tau_{k}^{(2)} y_{k+2}+\cdots+\tau_{k}^{(n+1)} y_{k+n+1} .
$$

The equation coincides with the Baxter's $T-Q$ relation for $n=1$ by putting $x=0$ and writing $y_{k}(x=0)=Q\left(\lambda q^{k+\text { const. }}\right)$. For general $n$, this coincides with the "spectral curve" equation argued in [46] or the characteristic equation in the quantum separation of variables 47.

\section{6 discussion}

We have seen that Stokes multipliers associated to a $n+1$ th differential equation satisfy the same recursion relations with fusion transfer matrices related to $U_{q}\left(A_{n}^{(1)}\right)$. Under the assumption on analyticity of some functions in the strip, we conjecture thermodynamic Bethe ansatz type equations eq.(46) which determine Stokes multipliers. The assumption , however, needs extensive numerical check, which we hope to report in the near future.

The deformation parameter $q=\exp \left(i \frac{2 \pi}{m+n+1}\right)$, which arises naturally in the present context, has a concrete meaning in the solvable models. The parameter $m$ specifies the level of dominant integral weight of the local variables in the lattice model 48]. Then the denominator of the exponent of $q$ is of the form, level + dual Coxeter number. This combination also appears as the deformation parameter of a solvable model based on other affine Lie algebras [49]. We thus expect a possible extension of the present study to other type of potential terms related to other affine Lie algebras.

\section{Acknowledgments}

The author thanks Y. Takei for discussions and comments. He also thanks A. Voros for comments and calling his attention to references [8, 9, 32].

\section{Appendix}

We present the argument leading to eq.(5)).

In terms of the vector $u:=\left(y, \partial y, \cdots, \partial^{n} y\right)$, eq.(2) reads,

$$
\frac{d}{d x} u=A(x) u=\left(\begin{array}{ccccc}
0, & 1, & 0, & \cdots, & 0 \\
0, & 0, & 1, & \cdots, & 0 \\
\vdots & & & & \vdots \\
0, & 0, & 0, & \cdots, & 1 \\
P(x), & 0, & 0, & \cdots, & 0
\end{array}\right)
$$


We define a new variable $\xi$ by $\xi^{n+1}=x$. Put $u=M T w$ such that $M=\operatorname{diag}\left(1, \xi^{m}, \xi^{2 m}, \cdots\right)$ and that $T$ diagonalizes a matrix of the form $A(x)$ in the above with $P(x)=1$. Then the equivalent matrix equation is written in the form

$$
\frac{d}{d \xi} w=\xi^{m+n} B(\xi) w=\xi^{m+n} \sum_{j=0} B_{j} \xi^{-j} w
$$

and $B_{0}=\operatorname{diag}\left(\exp \left(i \frac{2 \pi n}{n+1}\right), \exp \left(i \frac{2 \pi(n-1)}{n+1}\right), \cdots, 1\right)$.

Following [33, 34], we assume $w$ in the form,

$$
w=\left(\begin{array}{c}
p_{1}(\xi) \\
p_{2}(\xi) \\
\vdots \\
p_{n+1}(\xi)
\end{array}\right) \exp \left(\int^{\xi} \eta^{m+n} \gamma(\eta) d \eta\right)
$$

and $p_{1}(\xi)=1$

Then the substitution of the above form into eq. (48) yields,

$$
\xi^{-m-n} \frac{d}{d \xi} p_{j}=\beta_{j, 1}+\left(\beta_{j, j}-\beta_{1,1}\right) p_{j}-\sum_{i \neq 1} \beta_{1, i} p_{i} p_{j}+\sum_{k \neq 1, j} \beta_{j, k} p_{k}
$$

where $\beta_{j, k}$ denotes the $(i, j)$ th component of $B(\xi)$ and $j \geq 2$. Note that $\beta_{j, k}=\mathcal{O}(1 / \xi)$ if $j \neq k$.

Immediately seen, $\widehat{p_{j}}(\xi)=\sum_{N=1} p_{j, N} \xi^{-N}$ is a formal solution; one can recursively determine $p_{j, N}$ by (49).

We define $h_{0}=\beta_{n+1, n+1}(\infty)-\beta_{1,1}(\infty)$, and the sector $\mathcal{S}$ in the $\xi-$ plane by

$$
\left|\arg h_{0}+(m+n+1) \arg \xi\right| \leq \frac{3}{2} \pi, \quad|\xi| \geq \Omega
$$

for a fixed positive $\Omega$. By $\mathcal{D}_{r}$ we mean the domain in $\left(a_{1}, \cdots, a_{m}\right)$ space such that

$$
\left|a_{1}\right|^{2}+\cdots\left|a_{m}\right|^{2}<r
$$

for some positive $r$. Then we naturally generalize the result in [33, 34].

Conjecture 2 one can construct $\widehat{p_{j, r}}(\xi)$ so that $\widehat{p_{j, r}}(\xi)$ is holomorphic with respect to $\left(\xi, a_{1}, \cdots, a_{m}\right)$ in $\mathcal{S} \times \mathcal{D}_{r}$ and $\partial^{k} \widehat{p_{j, r}}(\xi),(k=0,1, \cdots)$ admits the uniformly asymptotic expansions,

$$
\partial^{k} \widehat{p_{j, r}}(\xi) \sim \partial^{k} \widehat{p_{j}}(\xi)
$$

for $\left(a_{1}, \cdots, a_{m}\right) \in \mathcal{D}_{r}$ as $\xi$ tends to infinity in $\mathcal{S}$. 
Let us present some argument supporting this (by no means a proof). We put $p_{j}=$ $q_{j}+\widehat{p_{j, r}}(\xi)$ and inserting this into (49),

$$
\begin{aligned}
\xi^{-m-n} \frac{d}{d \xi} q_{j}= & C_{j, 1}+C_{j, k} q_{j}-\sum_{i \neq 1} \beta_{1, i} q_{i} q_{j} \quad(j \geq 2), \\
C_{j, 1}= & \beta_{j, 1}+\left(\beta_{j, j}-\beta_{1,1}\right) \widehat{p_{j, r}}(\xi)+\sum_{k \neq 1, j} \beta_{j, k} \widehat{p_{k, r}}(\xi) \\
& -\sum_{i \geq 2} \beta_{1, i} \widehat{p_{i, r}}(\xi) \widehat{p_{j, r}}(\xi)-\xi^{-m-n} \frac{d}{d \xi} \widehat{p_{j, r}}(\xi) \quad(j \geq 2), \\
C_{j, k}= & \beta_{j, k}\left(1-\delta_{j, k}\right)+\delta_{j, k}\left\{\left(\beta_{j, j}-\beta_{1,1}\right)-\sum_{i \geq 2} \beta_{1, i} \widehat{p_{i, r}}(\xi)\right\} \\
- & \beta_{1, k} \widehat{p_{j, r}}(\xi), \quad(j, k \geq 2) .
\end{aligned}
$$

The formal solution is

$$
q_{j}(\xi)=\int^{\xi} \eta^{m+n}\left(C_{j, 1}+\phi_{j, k} q_{k}+\sum_{i \neq 1} \beta_{1, i} q_{i} q_{j}\right) \times \exp \left(\frac{h_{0}^{(j)}}{m+n+1}\left(\xi^{m+n+1}-\eta^{m+n+1}\right)\right) d \eta
$$

where we set $C_{j, k}=h_{0}^{(j)} \delta_{j, k}+\phi_{j, k}$ and $h_{0}^{(j)}=(n+1)\left(\exp \left(2 \pi i \frac{n+1-j}{n+1}\right)-\exp \left(2 \pi i \frac{n}{n+1}\right)\right)$. Now the coefficients in the bracket of the integrand are less than order $\frac{1}{\xi}$.

We are interested in eq.(1). For this, it may be enough to treat $j=n+1$. Assume there exists appropriate choice of $M$, then Lemma 3 of [33] also applies in this case. The integral in the rhs of (50) can be thus bounded from above, $\left|q_{n+1}\right| \leq \mathcal{O}\left(|\xi|^{-1}\right)$ in a certain domain provided that

$$
\left|\arg h_{0}^{(n+1)}+(m+n+1) \arg \xi\right|<\frac{3 \pi}{2}-\epsilon
$$

where $\epsilon$ is positive and small. By definition, $h_{0}^{(n+1)}=h_{0}$, thus in $\mathcal{S}, q_{n+1}$ tends to zero as $|\xi| \rightarrow \infty$. Hence the solution converges to the asymptotic expansion form. It may be shown that the limit does not depend on $r$ as in [33, 34]. The condition (51) coincides, when written in terms of $x$, with (5). 


\section{References}

[1] P. Dorey and R. Tateo, J. Phys. A 32 (1999) L419,(hep-th/9812211).

[2] P. Dorey and R. Tateo, "On the relation between Stokes multipliers and the $T-Q$ system of conformal field theory", (hep-th/9906219).

[3] A. Voros, Ann. Inst. H. Poincare A39 (1983) 211.

[4] A. Voros,Adv. Stud. Pure. Math. 21 (1992) 327.

[5] A. Voros, J. Phys. A27 (1994) 4653.

[6] A. Voros, in Quasiclassical Method (IMA Proceedings, Minneapolis 1995) eds. J.Rauch and B. Simon, IMA Series 95189 (Springer 1997).

[7] A. Voros, J. Phys. A 32 (1999) 1301.

[8] A. Voros, J. Phys. A 32 (1999) 5993.

[9] A. Voros, "Exact quantization method for the polynomial 1D Schrödinger equation", in Toward the exact WKB analysis of differential equations, linear or non-linear eds. T. Kawai et al. (Proceedings, Kyoto 1998), to be published by Kyoto University Press.

[10] T. Kawai and Y. Takei, Algebraic Analysis on Singular Perturbations (Iwanami, 1999) in Japanse.

[11] R. J. Baxter, Exactly Solved Models in Statistical Mechanics (Academic Press)

[12] V. V. Bazhanov, S. L. Lukyanov and A. B. Zamolodchikov, Comm. Math. Phys. 177 (1997) 381.

[13] V. V. Bazhanov, S. L. Lukyanov and A. B. Zamolodchikov, Comm. Math. Phys. 190 (1997) 247.

[14] P. Fendley, F. Lesage and H. Saleur, J. Stat. Phys. 85 (1996) 211.

[15] V. V. Bazhanov, S. L. Lukyanov and A. B. Zamolodchikov, Nucl. Phys. B489 (1997) 487.

[16] Al.B. Zamolodchikov, Phys. Lett B253 (1991) 391.

[17] A. Klümper and P. A. Pearce, Physica A183 (1992) 304.

[18] A. Klümper ,M.T. Batchelor and P. A. Pearce, J. Phys. A 24 (1991) 3111

[19] C. Destri and H.J. de Vega, Phys. Rev. Lett.69(1992) 2313.

[20] G. Jüttner, A. Klümper and J. Suzuki, Nucl. Phys. B487 (1997) 650. 
[21] G. Jüttner, A. Klümper A and J. Suzuki, Nucl. Phys. B 522(1998) 471 (condmat/9711310).

[22] J. Suzuki, J. Phys. A32(1999) 2341.

[23] J. Suzuki, J. Phys. A 32 (1999) L183,(hep-th/9902053).

[24] V. V. Bazhanov, S. L. Lukyanov and A. B. Zamolodchikov, "Spectral determinants for Schrödinger equation and $Q$ - operators of Conformal Field Theory", hepth/9812247.

[25] H. Scheffé, Trans. Amer. Math. Soc. 40 (1936) 127.

[26] H.L. Turrittin, Trans. Amer. Math. Soc. 68 (1950) 304.

[27] W.J. Trjitzinsky, Acta Math. 62 (1934) 167.

[28] V.V. Bazhanov and N. Yu Reshetikhin, J.Phys. A 23 (1990) 1477.

[29] A.Kuniba, T. Nakanishi and J. Suzuki, Int. J. Mod. Phys. A9 (1994) 5215, ibid 5267.

[30] P. Dorey and R. Tateo, "Differential equations and integrable models: $S U(3)$ case", hep-th/9910102.

[31] M. V. Fedoryuk, Aymptotic analysis (Springer 1993)

[32] A.B. Olde Daalhuis and F. W. J. Olver, SIAM Rev. 40 (1998) 463.

[33] P-F Hsieh and Y. Shibuya, J. Math. Analysis and Applications 16 (1966) 84.

[34] Y. Shibuya, Global Theory of second order linear ordinary differential operator with a polynomial coefficient Mathematics Studies 18 (North-Holland 1975)

[35] See, e.g., I. G. Macdonald, Symmetric functions and Hall polynomials, 2nd ed., Oxford University Press, 1995

[36] I. Cherednik, in Proc. of the XVII International Conference on Differential Geometric Methods in Theoretical Physics, Chester, ed. A.I. Solomon, (World Scientific, Singapore, 1989)

[37] A.N. Kirillov and N.Yu. Reshetikhin, Zap. Nauch. Semin. LOMI 160 (1987) 211 [J. Sov. Math. 52 (1990) 3156]

[38] A.Kuniba, Nucl. Phys. B389 209 (1993)

[39] A. Kuniba, Y.Ohta and J. Suzuki, J. Phys. A 28 (1995) 6211.

[40] J. Suzuki, Nucl Phys B528 (1998) 683.

[41] J. Suzuki, "Hidden E type structures in the dilute $A$ models" (hep-th/9909104). 
[42] P. A. Pearce and B. Nienhuis, Nucl Phys B519 (1998) 579.

[43] A. Klümper, Ann. Physik 1 (1992) 540.

[44] G. Jüttner, A. Klümper and J. Suzuki, Nucl Phys B512 (1998) 581.

[45] A. Kuniba, K. Sakai and J. Suzuki, Nucl Phys B525 597.

[46] B. Feigin and E. Frenkel, Comm. Math. Phys. 178 (1996) 653.

[47] E. Sklyanin, Prog. Theoret. Phys. 118 (1995) 35.

[48] M. Jimbo T. Miwa and M. Okado, Nucl. Phys, B300 (1988) 74.

[49] E. Date, M. Jimbo and M. Okado, Commun. Math. Phys. 155 (1993) 47. 